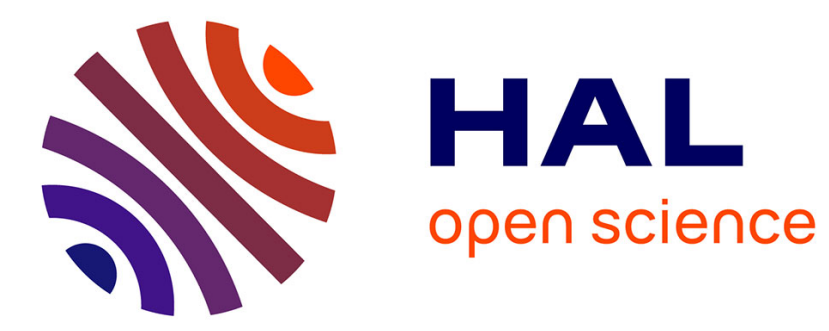

\title{
EFFET HALL DES LAMES MINCES DE NICKEL - PHOSPHORE
}

\author{
J. Flechon, M. Viard
}

\section{To cite this version:}

J. Flechon, M. Viard. EFFET HALL DES LAMES MINCES DE NICKEL - PHOSPHORE. Journal de Physique Colloques, 1968, 29 (C2), pp.C2-83-C2-86. 10.1051/jphyscol:1968214 . jpa-00213529

\section{HAL Id: jpa-00213529 https://hal.science/jpa-00213529}

Submitted on 1 Jan 1968

HAL is a multi-disciplinary open access archive for the deposit and dissemination of scientific research documents, whether they are published or not. The documents may come from teaching and research institutions in France or abroad, or from public or private research centers.
L'archive ouverte pluridisciplinaire HAL, est destinée au dépôt et à la diffusion de documents scientifiques de niveau recherche, publiés ou non, émanant des établissements d'enseignement et de recherche français ou étrangers, des laboratoires publics ou privés. 


\title{
EFFET HALL DES LAMES MINCES DE NICKEL - PHOSPHORE
}

\author{
J. Flechon et M. VIARD $\left(^{1}\right)$ \\ Laboratoire de Physique de dépôts métalliques, 54-Nancy
}

\begin{abstract}
Résumé. - Nous avons étudié l'effet Hall des films minces de nickel-phosphore obtenus par oxydo-réduction en phase liquide.

L'épaisseur varie de 300 à $1650 \AA$. Des recuits stabilisants, sous vide, permettent de modifier la structure donc les tensions observées.

Le comportement de ces couches minces est nettement celui des ferromagnétiques.

Notre étude a porté sur une centaine de lames minces de nickel-phosphore préparées à $20^{\circ} \mathrm{C}$ $[1-2]$.

Après séchage, ces films subissent avant toute mesure un recuit préalable correspondant à une stabilisation de la résistance [2]. Les mesures sont effectuées dans l'air et à la température ordinaire.

Abstract. - We have studied the Hall effect of the thin films of nickel-phosphorus derivedby oxido-reduction in liquid phase.

The thickness varies from $300 \AA$ à $1650 \AA$. Stabilising annealing under vacuum permits alteration of the structure; hence the stresses recorded. The behaviour of these thin layers is clearly that of the ferromagnetics.
\end{abstract}

Technique expérimentale. - Les dépôts $4 \mathrm{~cm} \times 1 \mathrm{~cm}$ sont réalisés sur lame de verre plan et répondent aux conditions optima permettant la mesure de la tension réelle [3].

Les contacts sont en nickel évaporé. L'effet Hall est mesuré en courant alternatif (BF. $1000 \mathrm{Hertz} ; 1 \mathrm{~mA}$ ) dans un champ d'induction $B$ continu et uniforme [4] pouvant varier de 0 à 2,5 teslas. Le montage est classique et couramment employé [5].

Un dispositif simple en courant continu avec mesure directe de la tension sur microvoltmètre, nous permet d'obtenir le signe de $V_{\mathbf{H}}$ et de comparer avec les valeurs précédentes.

La reproductibilité est de l'ordre de 5 à $8 \%$.

Aspect théorique. - Un grand nombre de théoriciens ont examiné le problème de l'effet Hall des ferromagnétiques. Leurs conclusions ne sont pas toujours en parfait accord.

Nous nous sommes attachés ici à une étude expérimentale de cet effet en examinant les points susceptibles de la rattacher aux théories existantes.

Pugh a traduit le phénomène par une loi expérimentale [6], exprimée ici dans le système M.K.S. A.

(1) Cet article recouvre en partie une thèse de Doctorat ès Sciences qui sera soutenue par M. Viard devant la Faculté des Sciences de Nancy. par

$\rho_{\mathrm{H}}=V_{\mathrm{H}} \cdot \frac{e}{i}=\mu_{0}\left(R_{0} H+R_{1} J\right)=R_{0} B+\mu_{0} R_{\mathrm{s}} J_{\mathrm{s}}$

avec

$V_{\mathrm{H}}=$ tension de Hall (volts), $e=$ épaisseur (m), $i=$ intensité du courant $(\mathrm{A}), \rho_{\mathbf{H}}=$ résistivité de Hall $(\Omega . \mathrm{m}), J=$ intensité d'aimantation $(\mathrm{A} / \mathrm{m}), R_{0}=$ constante ordinaire $\left(\mathrm{m}^{3} / \mathrm{C}\right), R_{1}=$ constante extraordinaire $\left(\mathrm{m}^{3} / \mathrm{C}\right), \quad R_{\mathrm{s}}=R_{1}-R_{0}$ coefficient spontané.

Les coefficients $R_{0}-R_{1}, R_{\mathrm{s}}$ sont déterminés à partir des pentes des droites de $\rho_{\mathrm{H}}$ et de leur intersection avec les axes [7], [8].

Influence du recuit sur la tension. - Nous avons pu noter la nécessité d'un recuit afin d'éliminer des fluctuations relativement importantes lors des mesures. Ceci étant relatif à un bruit de fond du dépôt [2].

Les températures de recuit $\left(50{ }^{\circ} \mathrm{C}-100^{\circ} \mathrm{C}-200{ }^{\circ} \mathrm{C}\right.$ $300^{\circ} \mathrm{C}-400^{\circ} \mathrm{C}$ ) sous un vide de $5 \times 10^{-6}$ torr, correspondent à l'apparition de phases mises en évidence par une étude cristallographique menée conjointement. Les temps de recuit sont en relation directe avec la stabilisation de la résistance du film [2]. 
La figure 1 traduit le comportement de 3 lames préparées dans les conditions précédentes. La polarité de $V_{\mathbf{H}}$ nous a permis de déceler une conductivité électronique.

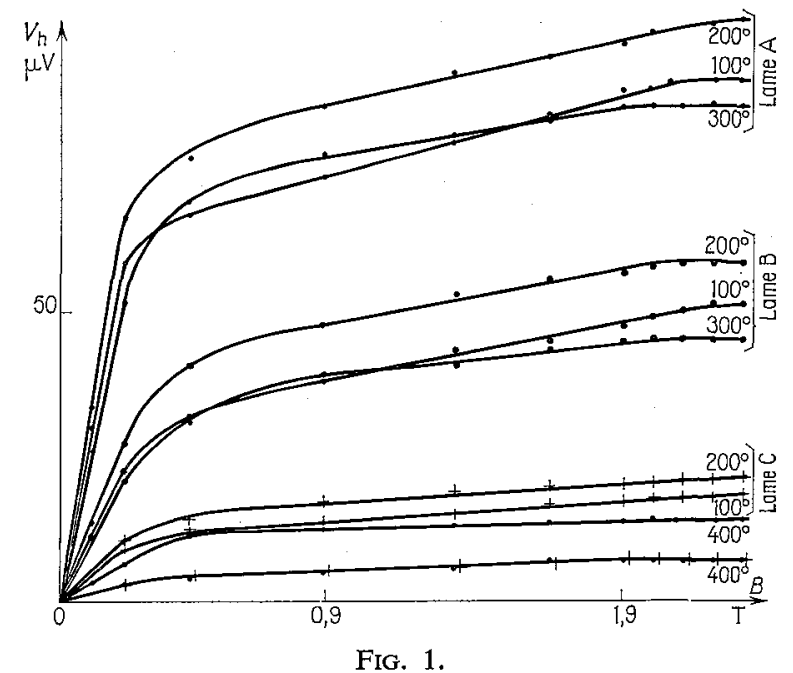

Nous observons une augmentation de la tension entre $100^{\circ} \mathrm{C}$ et $200^{\circ} \mathrm{C}$, puis l'effet décroît et tend après un recuit à $400^{\circ} \mathrm{C}$ vers une limite $(6 \mu \mathrm{V})$ voisine de celle du nickel pur pour les fortes épaisseurs (5b-9a). Cette décroissance avec le recuit a déjà été observée sur d'autres matériaux ferromagnétiques [10].

A $200^{\circ} \mathrm{C}$, l'étude aux rayons $\mathrm{X}$ de la substance révèle un début de cristallisation des phosphures de nickel dont les raies les plus intenses encadrent la raie (111) du nickel. L'augmentation de la tension de Hall à $200^{\circ} \mathrm{C}$ pourrait être expliquée par la formation progressive de ces phosphures [11].

$\mathrm{Au}$ delà de $300^{\circ} \mathrm{C}$, les raies (111) et (200) du nickel se séparent nettement de celles des phosphures comme le montrent les diagrammes aux rayons $\mathrm{X}$ et les clichés de microscopie électronique [1-11].

A partir de cette température de recuit, la résistivité des lames diminue fortement, de $28 \times 10^{-7} \Omega$.m à $4 \times 10^{-7} \Omega$.m pour les dépôts les plus épais. A ce moment, leur comportement serait voisin de celui du nickel pur évaporé et recuit dans les mêmes conditions [5].

Variation de la tension avec l'épaisseur. - Le phénomène est mis en évidence par les figures 2 et 3 relatives à des lames recuites à $200{ }^{\circ} \mathrm{C}$. Nous notons une variation normale de $1650 \AA$ à $550 \AA$, puis une décroissance à partir de $450 \AA$, anomalie déjà signalée [4].

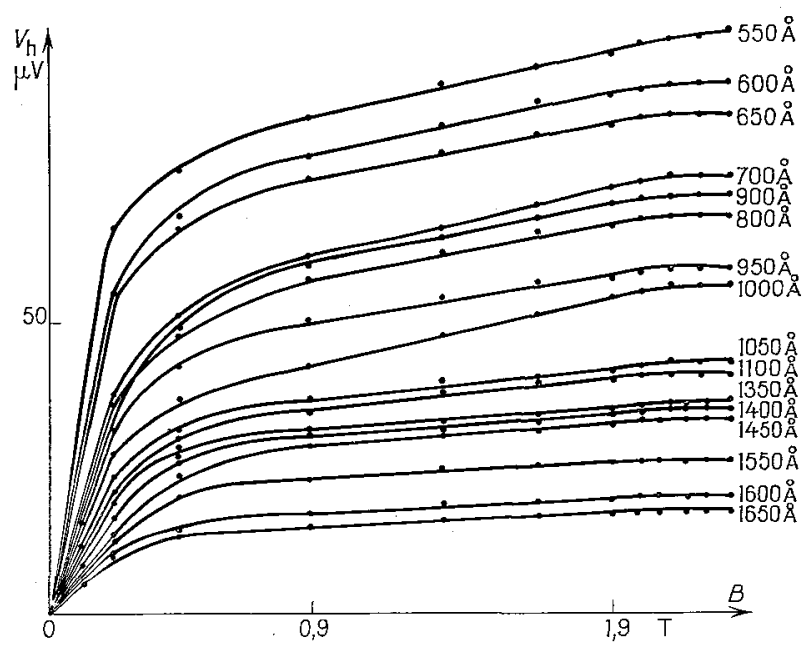

FIG. 2.

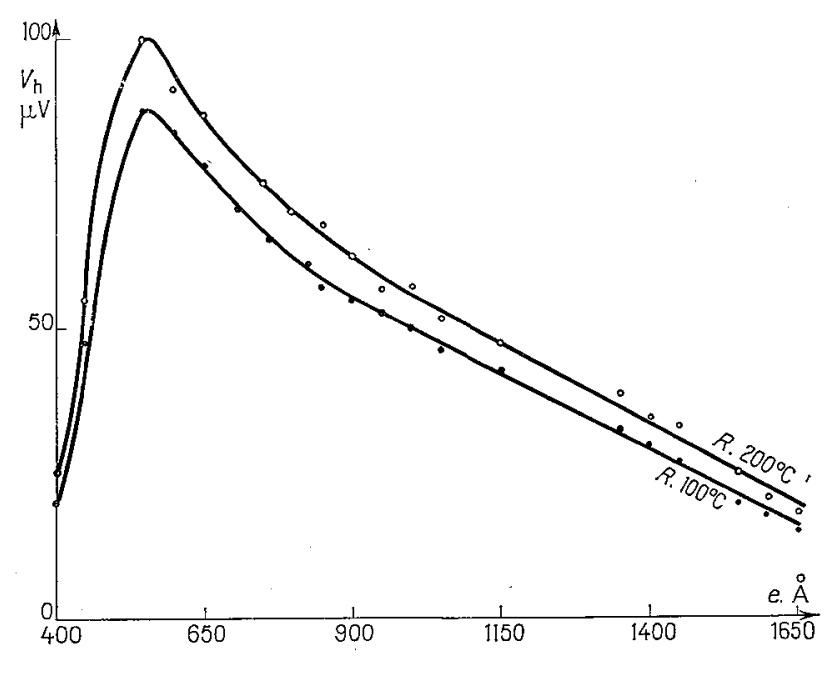

FIG. 3.

En effet, dans ce domaine d'épaisseur, le pourcentage en phosphore devient rapidement très grand [4] ainsi que la résistivité, $7000 \times 10^{-7} \Omega$.m pour une lame de $350 \AA$. L'effet Hall n'est plus alors mesurable ; il faut attendre l'apparition nette des phases du nickel (recuit $400^{\circ} \mathrm{C}$ ) pour observer à cette épaisseur un effet Hall significatif.

Détermination des constantes $R_{0}$ et $R_{1}$. - Elle s'effectue à partir du tracé des courbes $\rho_{\mathrm{H}}=f(B)$ (Fig. 4).

La figure 5 traduit la dépendance de ces grandeurs avec l'épaisseur et la température de recuit. Nous notons la présence d'un maximum au voisinage de 550 Å. La valeur de celui-ci diminue au fur et à mesure 


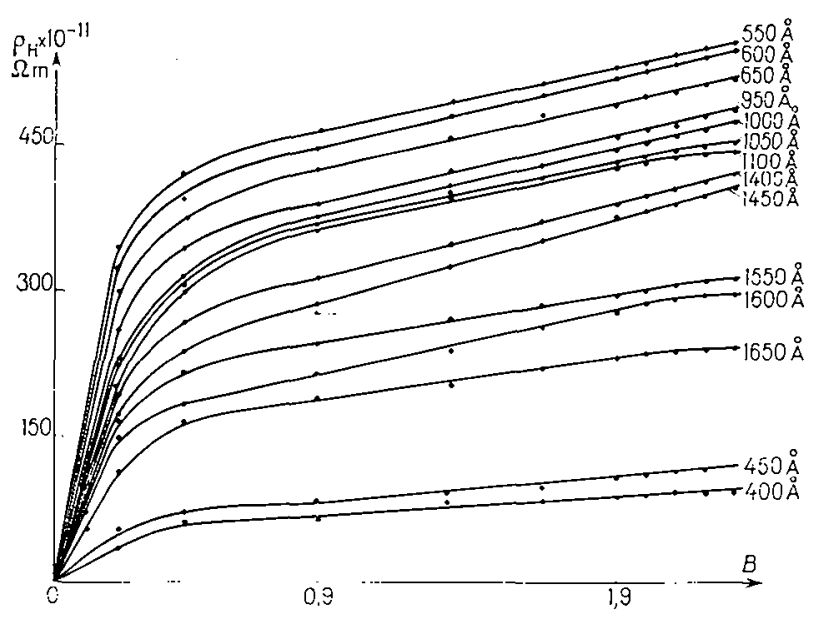

FIG. 4.

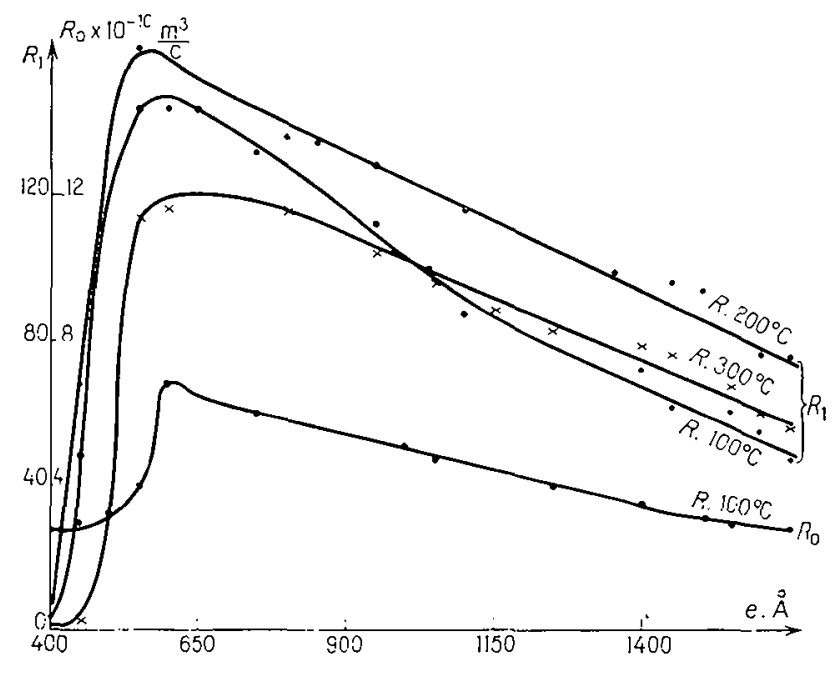

FIG. 5.

qu'augmente la température de recuit et le pic s'estompe après $400^{\circ} \mathrm{C}$ où la constante $R_{1}$ tend vers $15 \times 10^{-10} \mathrm{~m}^{3} / \mathrm{C}$ pour les fortes épaisseurs.

Dans les mêmes conditions, $R_{0}$ a pour limite

$$
0,3 \times 10^{-10} \mathrm{~m}^{3} / \mathrm{C} \text {. }
$$

Cette valeur nous permet de calculer le nombre $n$ d'électrons de conduction par atome.

$N=$ atomes par $\mathrm{m}^{3}, A=$ masse atomique du métal, $\rho_{0}=$ masse volumique.

$$
n=\frac{1}{R_{0}} \overline{N . e}=\frac{1}{R_{0}} \frac{A}{\rho_{0} \cdot e} .
$$

Soit à $400{ }^{\circ} \mathrm{C}: \quad n=2,28 \mathrm{e}^{-}$par atome.
Dans des conditions analogues mais sous vide, les expériences effectuées sur le nickel évaporé, conduisent à $R_{0}=0,6 \times 10^{-10} \mathrm{~m}^{3} / \mathrm{C}$ et $n=1,14$ [5]. Le phosphore semble donc jouer ici le rôle de ( dopeur ) comme dans le cas d'un semi-conducteur. Signalons également que compte tenu de la variation de $R_{0}$, le nombre de porteurs croît avec le recuit. Nous trouvons $n=1,27 \mathrm{e}^{-} /$atome après un recuit à $100^{\circ} \mathrm{C}$.

Les résultats précédents nous ont permis de tracer la variation du paramètre champ $\alpha=R_{1} / R$ en fonction de l'épalsscur pour un recuit à $100^{\circ} \mathrm{C}$ (Fig. 6).

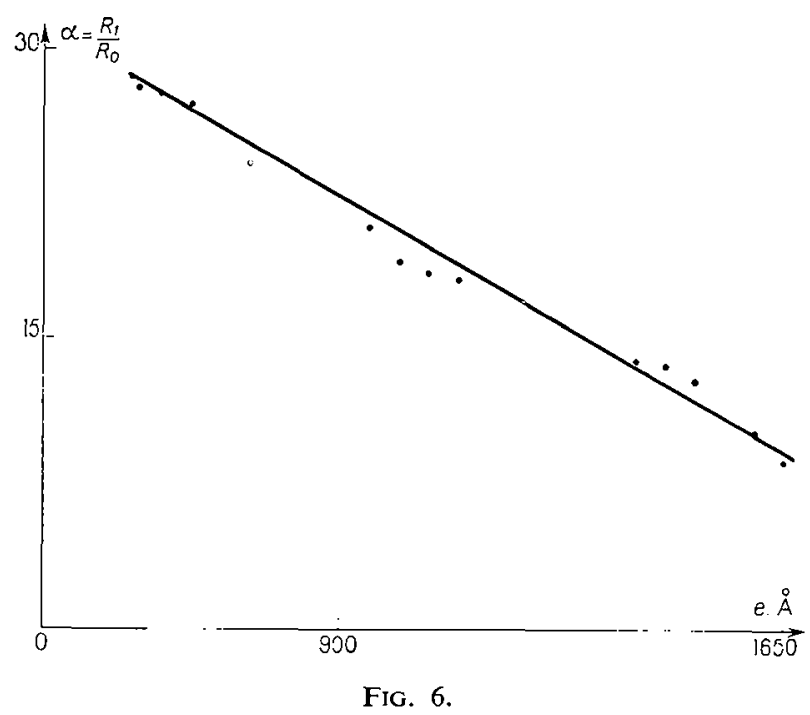

Nous obtenons une droite pour les épaisseurs comprises entre $500 \AA$ et $1650 \AA$; la variation est donc analogue à celles des autres corps ferromagnétiques [5-10]. La valeur élevée de ce paramètre n'a pas encore reçu d'explication valable.

Les points n'étant pas assez nombreux, il ne nous est pas possible de tracer la courbe $R_{\mathrm{s}}=A \rho^{n}$ ( $\rho$ résistivité du métal). Cependant, le tableau suivant nous permet

\begin{tabular}{|c|c|c|c|c|}
\hline $\begin{array}{l}\text { Recuit lame } \\
1600 \AA\end{array}$ & $100^{\circ} \mathrm{C}$ & $200^{\circ} \mathrm{C}$ & $300^{\circ} \mathrm{C}$ & $400^{\circ} \mathrm{C}$ \\
\hline$\rho . \Omega . m$ & $18 \times 10^{-}$ & $14 \times 10$ & $8,8 \times 10^{-7}$ & $4 \times 10^{-7}$ \\
\hline$R_{\mathrm{s} \mathrm{m}}{ }^{3} / \mathrm{C}^{1} 0^{-9}$ & 7,24 & 5,56 & 5 & 1,2 \\
\hline$n_{\mathfrak{e}-}$ & 1,6 & 1,55 & 1,48 & 1,45 \\
\hline Lame $1000 \AA$ & $n=1,8$ & 2,15 & 1,85 & 1,43 \\
\hline Lame $650 \AA$. & $n:=1,7$ & 1,48 & 1,48 & 1,40 \\
\hline
\end{tabular}
d'indiquer quelques conclusions. 
La théorie de Karpluss et Luttinger [12] prévoit, en effet, une loi de la forme $R_{\mathrm{s}}=A \rho^{n}$ avec $n=2$. Or, les travaux de Jan et Gyssman [13] Butler et Pugh [14] sur le nickel, ont conduit à poser $n=1,42$.

Karpluss et Luttinger ont été alors amenés à reviser leur théorie qui fut également reprise par Smith [15]. Ces théoriciens admirent que le coefficient $n$ pouvait être compris entre 1,4 et 2. Dans nos expériences, $n$ supérieur à 1,4 pour les premiers recuits, tend vers cette dernière valeur après passage à $400^{\circ} \mathrm{C}$. Ceci confirme donc les résultats des paragraphes précédents.

Conductivité de Hall. - Les hypothèses émises par Pcrrier [16] nous conduisent à étudier les variations de la conductivité de Hall spontanée;

$$
\gamma_{H \mathrm{~s}}=\rho_{H \mathrm{~s}} / \rho^{2}
$$

et de l'angle d'anisotropie $\varphi_{H \mathrm{~s}}=\rho_{\mathrm{Hs}} / \rho$.

Les figures 7 et 8 nous donnent respectivement l'allure des phénomènes observés.

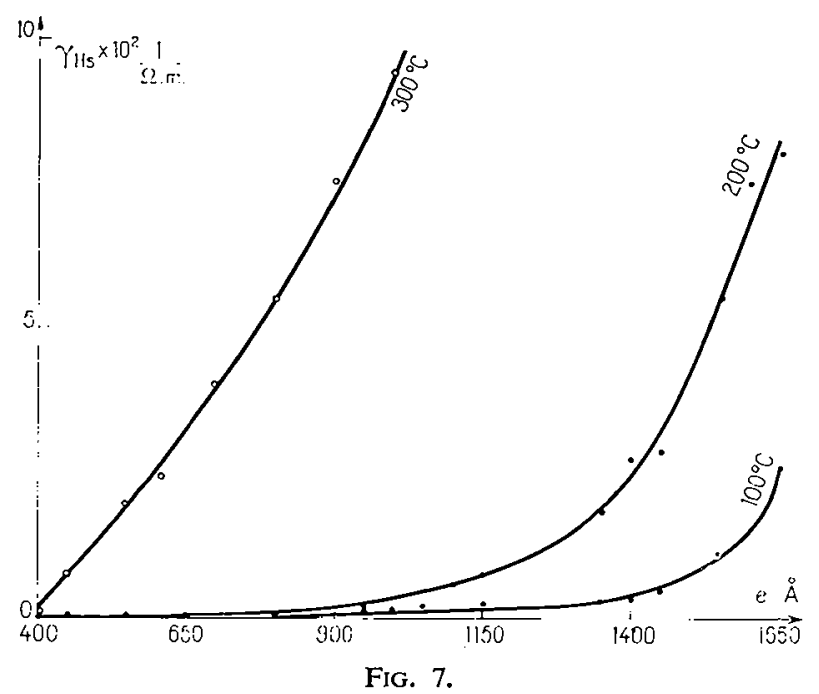

La conductivité de Hall $\gamma_{H \mathrm{~S}}$ croît avec la température de recuit. Après un recuit à $300^{\circ} \mathrm{C}$, la courbe $\gamma_{H s}$ traduit une augmentation particulièrement importante de ce paramètre. Ce phénomène confirme l'apparition des phases du nickel déjà mise en évidence dans les expériences précédentes. Les valeurs de $\gamma_{H \mathrm{~s}}$ et $\varphi_{H \mathrm{~s}}$ relatives aux premières températures de recuit $\left(100^{\circ} \mathrm{C}-200^{\circ} \mathrm{C}\right)$ sont nettement inférieures à celles du

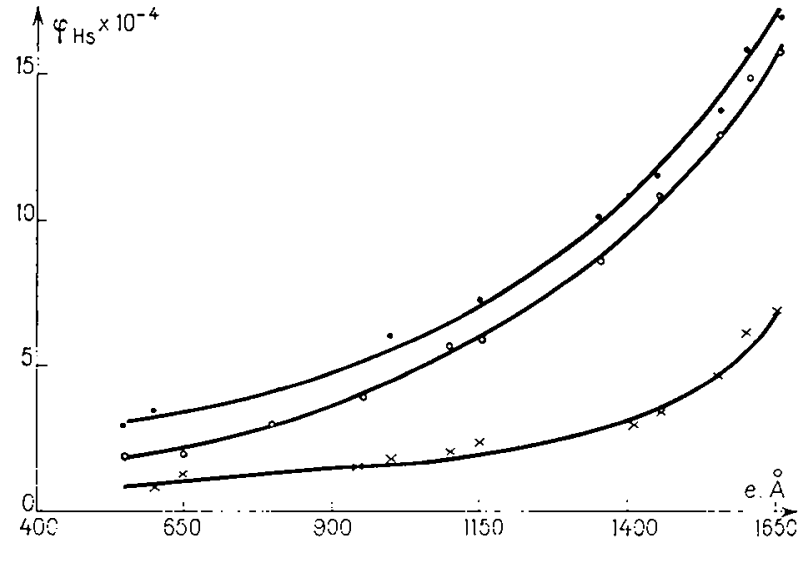

FIG. 8.

nickel massif et des alliages nickel-fer; mais elles s'en rapprochent après recuits à $300^{\circ} \mathrm{C}$ et $400^{\circ} \mathrm{C}$ [8-13].

Conclusion. - Le phénomène de Hall observé sur les films minces de nickel-phosphore montre que ce corps se comporte comme un ferromagnétique. De plus, les résultats obtenus entrent dans le cadre des prévisions théoriques et permettent, en fonction de la température de recuit, de considérer le dépôt comme un intermédiaire entre le nickel pur et le fer.

\section{Bibliographie}

[1] FléCHON (J.), Thèse Fac. Sc. Nancy, 1960.

[2] Machizaud (F.), Thèse $3^{e}$ cycle Fac. Sc. Nancy, 1965.

[3] JAN (J. P.), Thèse Fac. Sc. Lausanne, 1952, 679, 700.

[4] Viard (M.), D. E. S. Nancy, 1965.

[5] Goureaux (G.), Thèse Fac. Sc. Caen, 1960.

[6] Pugh (E. M.), Phys. Rev., 1930, 36, 1503.

[7] Corren (R.), Juretschke (J.), J. Appl. Physics, 1957, 23, 7.

[8] Ascher (E. A.), Helv. physica Acta, 1955, 12, VIII, 671-673.

[9] Rfimer (L.), Z. Physik, 1958, Bd 150, 280.

[10] Vautier, $J$. Physique, 1966, 27, 532.

[11] Machizaud (F.), Fléchon (J.), C. S. Ac. Sc. Paris, $1966,263,1500$.

[12] Karpluss, Luttinger, Phys. Rev., 1954, 21, 1158.

[13] Jan (J. P.), Gyssmann (J.), Physica, 1952, 18, 339.

[14] Butler et Pugh, Phys. Rev., 1940, 57, 916.

[15] Sмit (J.), Physica, 1955, 21, 877. Smit (J.), Nuovo Cimento, 1957, 3, 1177.

[16] Perrier, Helvetica, Phys. Acta, 1930, 3, 400. 